\title{
GESTÃO DOS PARÂMETROS DE ESTOQUE: ESTUDO DE CASO DE UMA EMPRESA VAREJISTA DE AUTO PEÇAS
}

\author{
Marina Ferreira Rodrigues - marinaengprod@gmail.com \\ Mário César Fialho de Oliveira - mc-mariocesar@hotmail.com \\ Douglas Barbosa-douglas.barbosa@professor.unis.edu.br \\ Márcia Marazo Pinto - marcia.marazo@professor.unis.edu.br \\ Patrícia Werneck Silva de Oliveira - paticso@yahoo.com.br
}

\section{RESUMO}

O objetivo dessa pesquisa é apresentar novos parâmetros de reposição de estoque de produtos de maior faturamento, especificamente as Bobinas de Ignição. Na Metodologia, foram objetos de estudo, 31 famílias de produtos, que são considerados itens de estoque, que estão disponíveis para venda na empresa estudada. Para tanto, utilizou-se do Teorema de Pareto para classificar a família que representa maior valor de faturamento no mês de Agosto de 2020, então estudou-se um grupo de 25 itens do estoque da família das Bobinas de Ignição. A partir daí, com base na filosofia de LEC (Lote Econômico de Compra) foram apresentados novos parâmetros de reposição de estoques. Os resultados encontrados na pesquisa mostram que a empresa apresenta uma parametrização de reposição de estoques falha, ficou evidenciado que a empresa, desde o início de suas operações, fez um investimento na compra de Bobinas de Ignição no valor total de $\mathrm{R} \$ 10.552,25$, porém, se utilizasse corretos parâmetros de reposição de estoque o valor total investido seria de $\mathrm{R} \$ 6.709,94$, sendo assim possível analisar que o valor investido foi mais do que o necessário desde sua abertura, excedendo cerca de $\mathrm{R} \$ 3.842,31$ na compra de Bobinas de Ignição.

Palavras-Chave: Gestão de Estoques, Teorema de Pareto, Lote Econômico de Compra. 


\section{INTRODUÇÃO}

Um sistema de controle de estoque é fundamentalmente, um conjunto de regras e procedimentos que permite responder a algumas perguntas e tomar algumas decisões sobre estoques (MOREIRA, 1993). O desafio do gestor de estoques é saber quando e quanto ressuprir de cada material e quanto deve manter em estoque de segurança. Com o crescente número de itens com diferentes padrões de demanda e características específicas, a complexidade na administração de materiais aumenta devido à necessidade de um controle diferenciado. (SANTOS; RODRIGUES, 2006)

Um dos principais motivos para se ter um bom planejamento e controle de estoques é o grande impacto financeiro que é possível alcançar através do aumento da eficácia e eficiência das operações da organização (BORGES et al, 2010). O objetivo é reduzir a quantidade de estoque a um nível mais baixo possível, consistente com as metas de prestação de serviço ao cliente de modo a obter o menor custo logístico total (BOWERSOX, 2001, pg $50)$.

O Lote Econômico de Compra (LEC) desenvolve um conceito de estoque ideal e é dividido em dois estudos inter-relacionados, os custos de armazenamento de estoque, que incluem os custos de capital empatado, armazenagem, obsolescência, e os de pedidos, evidenciando os custos nos descontos dos preços, transporte dos empregados e fornecedores (SLACK; CHAMBERS; JOHNSTON, 2009).

Com isso, sendo fundamental um controle eficiente do estoque, o presente estudo, feito através de uma pesquisa-ação e revisão bibliográfica sobre o tema por meio de livros e artigos científicos, tem por objetivo fornecer a empresa de Auto Peças parâmetros de reposições de estoque a fim de que estas possam ser feitas adequadamente, sem excessos de produtos adquiridos indevidamente. 


\section{REVISÃO DE LITERATURA}

\subsection{Gestão de Estoque}

Presumindo ter o entendimento do que é a gestão de estoques, é essencial, inicialmente conhecer qual a definição de estoque. "Os estoques existem devido a uma inadequação entre suprimento e demanda e, em lojas varejistas, essa inadequação pode ser proposital, pois é preferível manter um estoque como forma de antecipação a uma futura demanda" (CHOPRA; MEINDL, 2003). De acordo com Souza (2010) os estoques, como outros tipos de ativos, representam custos relevantes para as empresas e eficiência de sua gestão torna-se fator fundamental de competitividade.

Sendo assim, segundo Dias (2012) a Gestão de Estoques pode ser definida como o planejamento e controle de mercadorias para uma ligeira reposição, contando desde sua entrada, até a sua saída. E para que isso possa ocorrer, o gerente de estoques deve estar atento as seguintes situações: 1) Redução ou crescimento da rotatividade de mercadorias; 2) Aumento ou diminuição dos custos dos produtos estocados e da obsolescência; 3) Variação das vendas em virtude da estocagem de produtos realmente necessários.

“A própria definição de gestão de estoques evidencia seus objetivos, que são essencialmente, planejar o estoque, as quantidades de materiais que entram e saem, as épocas em que ocorrem as entradas e saídas, o tempo que decorrem entre essas épocas e os pontos de pedidos" (CHING, 2010).

Para gerir bem um estoque, os níveis de armazenagem devem ser os mais baixos
possíveis sem que haja um déficit para atender a demanda dos clientes. Para isso é
necessário que exista um controle de cada produto observando sua rotatividade, o
valor do produto e se há espaço suficiente para o armazenamento (BALLOU, 2007).

"É crescente a importância dada à gestão de estoques, sendo essa fundamental na redução e controle dos custos operacionais de uma empresa, bem como na melhoria de seus serviços prestados" (WANKE, 2003). Ballou (2001), apresenta os benefícios que a empresa alcança com a gestão dos estoques, que são: a melhoria dos serviços de atendimento ao consumidor; os estoques agem como amortecedores entre o suprimento e a demanda; podem conceder economia de escala nas compras e; atuam como proteção contra contingências e aumento de preços.

"Além disso existem também algumas dificuldades na gestão eficaz de estoques. Uma das maiores dificuldades da gestão da cadeia de suprimentos é o gerenciamento eficaz dos estoques, pois ele exige uma coordenação de todas as atividades e ligações na cadeia de suprimento" (TURBAN; RAINER; POTTER, 2003). 


\section{METODOLOGIA}

\subsection{Coleta de Dados}

Sabe-se que o controle e gerenciamento de estoques multi-itens é bastante complexo devido à diversidade. No entanto, para efeito de simplificação deste estudo, focou-se os itens chamados de alto valor de faturamento. Os dados para realização deste estudo foram disponibilizados através do Software utilizado pela empresa que é o Omie, ERP (Enterprise Resource Planning), permitindo acessar busca de dados, planilhas e relatórios necessários para pesquisa, e, também, algumas ferramentas como o Microsoft Excel, que se tratando de uma ferramenta mais usual e de fácil acesso, foi fundamental para a pesquisa, uma vez que com ele foi possível analisar os dados, criar, exibir e editar planilhas e tabelas, além de manipulá-los mais facilmente.

\subsection{Teorema de Pareto}

Após a análise dos dados coletados, com o objetivo de priorizar os itens que demandam maior atenção por parte da empresa, foi utilizado o Teorema de Pareto, que de acordo com Pozo (2004) é a lei fundamentada em um estudo sobre riqueza e renda, onde constatou-se que na minoria da população $(20 \%)$ se concentrava a maior parte da riqueza (80\%), daí o nome princípio de Pareto ou lei do 80/20.

A partir disso, de acordo com a quantidade movimentada no mês de Agosto, os itens foram classificados por famílias e logo após classificados em ordem decrescente de faturamento.

Os produtos classificados na primeira família, são os que proporcionam maior faturamento para a empresa, e por conta disso nunca podem faltar. Então, utilizando a primeira família classificada no Teorema de Pareto serão feitos os cálculos que serão demonstrados a seguir.

\subsection{Lote Econômico de Compra (LEC)}

Em seguida calculou-se o Ponto de Pedido e o Estoque de Segurança da primeira família classificada para que o Lote Econômico de Compras (LEC) dela fosse encontrado. O Lote Econômico de Compras (LEC) [...] busca a quantidade econômica mais eficaz em seus estoques, através de pedidos solicitados para os fornecedores de maneira rentável, indicando os períodos de solicitações de pedidos mais concretos e consecutivamente, disponibilizando um maior controle do sistema de armazenagem (BITENCOURT ECAVALCANTE, 2013). 
Para se calcular o Lote Econômico de Compras foi usada a seguinte Equação 01:

$$
\sqrt{\frac{2 * D m * C_{P}}{C m * C M C}}
$$

Fonte: A Autora (2020)

Onde Dm $=$ Demanda Mensal

$\mathrm{Cp}=$ Custo de Processamento de Pedidos

$\mathrm{Cm}=$ Custo de Manutenção de Estoques

$\mathrm{CMC}=$ Custo Médio Contábil (Unitário)

\section{RESULTADOS E DISCUSSÃO}

De acordo com as informações da gerência e as observações realizadas durante a pesquisa de campo, primeiramente foi feito o Gráfico de Pareto que, neste caso, buscou analisar qual família obteve maior faturamento no mês de Agosto de 2020.

\section{Gráfico (01) Faturamento por Família de Produtos (Teorema de Pareto)}

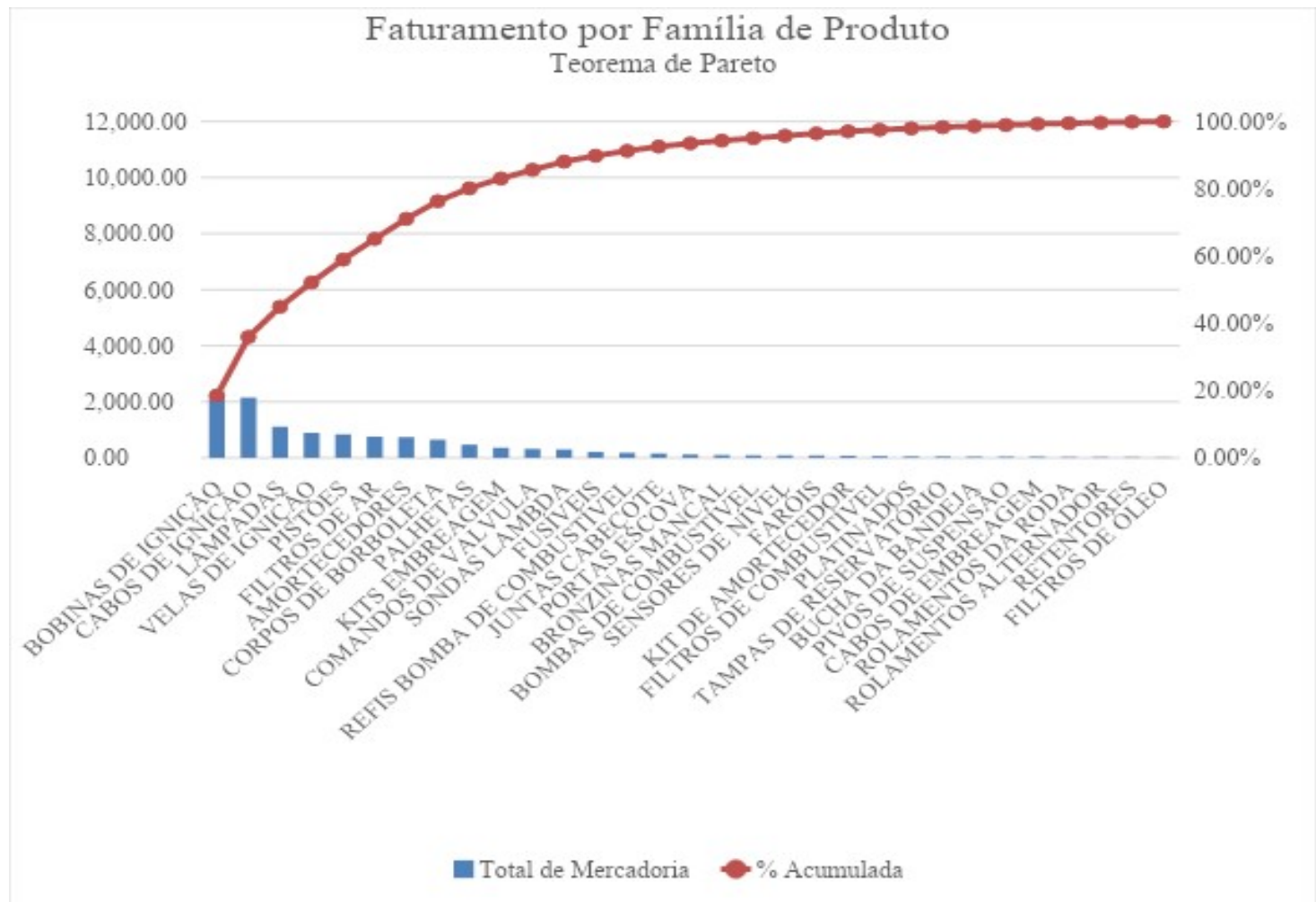

Fonte: A Autora (2020)

Para construir o gráfico foram utilizados os dados de venda do mês de Agosto de 2020 de cada família, sendo eles inseridos, em reais, em uma planilha de Excel, como pode-se ver 
no Apêndice 01, e depois colocados em ordem crescente de valores. A partir daí foram levantadas as porcentagens de vendas de cada família em relação ao total de vendas do mês em questão e também a porcentagem acumulada destas, onde soma-se a porcentagem anterior com a atual sucessivamente até que se complete o total de $100 \%$.

De acordo com o Gráfico de Pareto foi possível analisar que a família de Bobinas de Ignição foi a que obteve o maior faturamento do mês de Agosto de 2020, representando cerca de $18,5 \%$ do faturamento total do mês, sendo essa, portanto, escolhida para ser analisada nos cálculos posteriores.

Logo após a análise foram feitos os cálculos do Lote Econômico de Compra como pode-se ver na Tabela 01 .

Tabela (01) Cálculo Lote Econômico de Compra (LEC)

\begin{tabular}{|c|c|c|c|c|c|c|c|c|c|c|}
\hline \multicolumn{11}{|c|}{ Cálculo Lote Econômico de Compra (LEC) } \\
\hline $\begin{array}{c}\text { Código } \\
\text { do } \\
\text { Produto }\end{array}$ & Descrição do Produto & $\begin{array}{c}\text { Demanda } \\
\text { Mensal }\end{array}$ & & $\begin{array}{l}\text { MC } \\
\text { itário }\end{array}$ & & $\begin{array}{l}\text { le } \\
\text { ento } \\
\text { los }\end{array}$ & $\begin{array}{r}\text { C } \\
\text { Mar } \\
\text { de } \\
\end{array}$ & $\begin{array}{l}\text { o de } \\
\text { enção } \\
\text { toque }\end{array}$ & LEC & $\begin{array}{c}\text { LEC } \\
\text { (Arredondamento) }\end{array}$ \\
\hline 70 & BOBNNA IGNICAO BI0013MM & 0 & RS & 146,58 & RS & 3,04 & RS & 10,38 & 0 & 0 \\
\hline 71 & BOBNA IGNICAO BI0014MM & 0,25 & RS & $\$ 5,47$ & RS & 3,04 & RS & 10,38 & 4 & 4 \\
\hline 72 & BOBNA IGNICAO BI0017MM & 1,375 & RS & 140,84 & RS & 3,04 & RS & 10,38 & 10,65034351 & 11 \\
\hline 73 & BOBNA IGNICAO BI0051MM & 0 & RS & 104,65 & RS & 3,04 & RS & 10,38 & 0 & 0 \\
\hline 74 & BOBINA IGNICAO BI0079MM & 0 & RS & 95,47 & RS & 3,04 & RS & 10,38 & 0 & 0 \\
\hline 75 & BOBNA IGNICAO BI0101MM & 0 & RS & 114,10 & RS & 3,04 & RS & 10,38 & 0 & 0 \\
\hline 76 & BOBLNA IGNICAO BI0021MM & 0,125 & RS & 50,57 & RS & 3,04 & RS & 10,38 & 1,924219432 & 2 \\
\hline 78 & BOBNA IG.ICAO US0\$1 & 0 & RS & 137,36 & RS & 3,04 & RS & 10,38 & 0 & 0 \\
\hline 79 & BOBNA IGNICAO U2008 & 0,375 & RS & 121,62 & RS & 3,04 & RS & 10,38 & 5,168473797 & 6 \\
\hline 80 & BOBINA IGNICAO U3001 & 0,25 & RS & 43,02 & RS & 3,04 & RS & 10,38 & 2,50991099 & 3 \\
\hline 81 & BOBNA IGNICAO CE20119 & 0 & RS & 78,97 & RS & 3,04 & RS & 10,38 & 0 & 0 \\
\hline 82 & BOBNA IGNICAO F000ZS0235 & 0 & RS & 96,32 & RS & 3,04 & RS & 10,38 & 0 & 0 \\
\hline 87 & BOBNA IGNICAO BI0020MM & 0 & RS & 78,25 & RS & 3,04 & RS & 10,38 & 0 & 0 \\
\hline 88 & BOBNA IGNICAO 0780002010 & 0,875 & RS & 47,05 & RS & 3,04 & RS & 10,38 & 4,910627067 & 5 \\
\hline 97 & BOBNA IGNICAO U6043 & 0 & RS & 253,76 & RS & 3,04 & RS & 10,38 & 0 & 0 \\
\hline 129 & BOBLNA IGNICAO U1093 & 0 & RS & 80,80 & RS & 3,04 & RS & 10,38 & 0 & 0 \\
\hline 141 & BOBNA IGNICAO BI0060MM & 0,25 & RS & 253,97 & RS & 3,04 & RS & 10,38 & 6,098377813 & 7 \\
\hline 147 & BOBLNA IGNICAO U5145 & 0,125 & RS & 170,16 & RS & 3,04 & RS & 10,38 & 3,529692384 & 4 \\
\hline 153 & BOBINA IGNICAO B10024MM & 0,125 & RS & 46,09 & RS & 3,04 & RS & 10,38 & 1,837009801 & 2 \\
\hline 159 & BOBNA IGNICAO BI0040MM & 0 & RS & 181,77 & RS & 3,04 & RS & 10,38 & 0 & 0 \\
\hline 162 & BOBLNA IGNICAO U1054 & 0 & RS & 47,07 & RS & 3,04 & RS & 10,38 & 0 & 0 \\
\hline 177 & BOBINA IGNICAO CE10105 & 0 & RS & 41,89 & RS & 3,04 & RS & 10,38 & 0 & 0 \\
\hline 208 & BOBINA IGNICAO EBI9127 & 0 & RS & 131,80 & RS & 3,04 & RS & 10,38 & 0 & 0 \\
\hline 340 & BOBNA IGNICAO BI0105MM & 0,5 & RS & 153,30 & RS & 3,04 & RS & 10,38 & 6,700530563 & 7 \\
\hline 460 & BOBNA IGNICAO BI0114MM & 0 & RS & 155,79 & RS & 3,04 & RS & 10,38 & 0 & 0 \\
\hline
\end{tabular}

Fonte: A autora (2020)

Para efetuar essa tabela foi utilizada a fórmula do LEC (Lote Econômico de Compra) demonstrada anteriormente nas referências bibliográficas onde a raiz de, "dois abre parênteses", dois multiplicado a Demanda Mensal (Dm), multiplicado pelo Custo De Processamento (Cp), "fecha parênteses", dividido pelo "abre parênteses" Custo de 
Manutenção de Estoque $(\mathrm{Cm})$ multiplicado pelo Custo Médio Unitário (CMC) "dois fecha parênteses" é igual ao resultado do LEC.

Feito esses cálculos para todos os 25 códigos diferentes de Bobinas foi possível analisar que 15 Bobinas de Ignição têm o LEC iguais a zero, pois nunca tiveram saída. Por isso não haveria o porquê repor, se o histórico mostra que não há demanda. Já as outras 10 Bobinas que tiveram saída variaram o LEC entre 2 e 11.

A partir desses resultados foi possível fazer a análise comparativa de quanto a empresa investiu na compra e quanto ela deveria ter investido na família de Bobinas de Ignição, como pode ser visto na Tabela 02 a seguir:

Tabela (02) Análise Comparativa

\begin{tabular}{|c|c|c|c|c|c|c|c|c|c|c|}
\hline \multicolumn{11}{|c|}{ Análise Comparativa } \\
\hline \multirow{2}{*}{$\begin{array}{c}\text { CMC } \\
\text { Unitário } \\
R \$ 146,58\end{array}$} & \multirow{2}{*}{$\begin{array}{c}\text { LEC } \\
0 \\
\end{array}$} & \multirow{2}{*}{$\begin{array}{c}\begin{array}{c}\text { Quantidade } \\
\text { Vendida }\end{array} \\
0 \\
\end{array}$} & \multirow{2}{*}{$\begin{array}{c}\text { Pedidos de } \\
\text { Compra com } \\
\text { Fornecedor } \\
0 \\
\end{array}$} & \multirow{2}{*}{\begin{tabular}{|c}
$\begin{array}{c}\text { Quant. Média } \\
\text { Comprada desde a } \\
\text { abertura da firma }\end{array}$ \\
1 \\
\end{tabular}} & \multicolumn{2}{|c|}{$\begin{array}{c}\text { Gasto para } \\
\text { estoque correto } \\
\text { (R\$) }\end{array}$} & \multicolumn{2}{|c|}{$\begin{array}{l}\text { Valor Médio } \\
\text { Investido em } \\
\text { Estoque (R\$) }\end{array}$} & \multicolumn{2}{|c|}{ Resultado (R\$) } \\
\hline & & & & & $\mathrm{R} \$$ & - & $\mathrm{R} \$$ & 146,58 & $\mathrm{R} \$$ & 146,58 \\
\hline $\mathrm{R} \$ 85,47$ & 4 & 2 & 4 & $\begin{array}{l}7 \\
\end{array}$ & $\mathrm{R} \$$ & 341,88 & $\mathrm{R} \$$ & 598,29 & $\mathrm{R} \$$ & 256,41 \\
\hline $\mathrm{R} \$ 140,84$ & 11 & 11 & 11 & 22 & $\mathrm{R} \$$ & $1.549,21$ & $\mathrm{R} \$$ & $3.098,43$ & $\mathrm{R} \$$ & $1.549,21$ \\
\hline $\mathrm{R} \$ 104,65$ & 0 & 0 & 0 & 1 & $\mathrm{R} \$$ & - & $\mathrm{R} \$$ & 104,65 & $\mathrm{R} \$$ & 104,65 \\
\hline $\mathrm{R} \$ 95,47$ & 0 & 0 & 0 & 1 & $\mathrm{R} \$$ & - & $\mathrm{R} \$$ & 95,47 & $\mathrm{R} \$$ & 95,47 \\
\hline $\mathrm{R} \$ 114,10$ & 0 & 0 & 0 & 2 & $\mathrm{R} \$$ & - & $\mathrm{R} \$$ & 228,20 & $\mathrm{R} \$$ & 228,20 \\
\hline $\mathrm{R} \$ \quad 50,57$ & 2 & 1 & 2 & 6 & $\mathrm{R} \$$ & 101,14 & $\mathrm{R} \$$ & 303,42 & $\mathrm{R} \$$ & 202,28 \\
\hline $\mathrm{R} \$ 137,36$ & 0 & 0 & 0 & 1 & $\mathrm{R} \$$ & - & $\mathrm{R} \$$ & 137,36 & $\mathrm{R} \$$ & 137,36 \\
\hline $\mathrm{R} \$ 121,62$ & 6 & 3 & 6 & 7 & $\mathrm{R} \$$ & 729,69 & $\mathrm{R} \$$ & 851,31 & $\mathrm{R} \$$ & 121,62 \\
\hline $\mathrm{R} \$ \quad 43,02$ & 3 & 2 & 3 & 6 & $\mathrm{R} \$$ & 129,06 & $\mathrm{R} \$$ & 258,12 & $\mathrm{R} \$$ & 129,06 \\
\hline$R \$ \quad 78,97$ & 0 & 0 & 0 & 2 & $\mathrm{R} \$$ & - & $\mathrm{R} \$$ & 157,94 & $\mathrm{R} \$$ & 157,94 \\
\hline $\mathrm{R} \$ 96,32$ & 0 & 0 & 0 & 2 & $\mathrm{R} \$$ & - & $\mathrm{R} \$$ & 192,64 & $\mathrm{R} \$$ & 192,64 \\
\hline $\mathrm{R} \$ 78,25$ & 0 & 0 & 0 & 1 & $\mathrm{R} \$$ & - & $\mathrm{R} \$$ & 78,25 & $\mathrm{R} \$$ & 78,25 \\
\hline $\mathrm{R} \$ \quad 47,05$ & 5 & 7 & 10 & 14 & $\mathrm{R} \$$ & 235,25 & $\mathrm{R} \$$ & 658,70 & $\mathrm{R} \$$ & 423,45 \\
\hline$R \$ 253,76$ & 0 & 0 & 0 & 1 & $\mathrm{R} \$$ & - & $\mathrm{R} \$$ & 253,76 & $\mathrm{R} \$$ & 253,76 \\
\hline $\mathrm{R} \$ \quad 80,80$ & 0 & 0 & 0 & 2 & $\mathrm{R} \$$ & - & $\mathrm{R} \$$ & 161,60 & $\mathrm{R} \$$ & 161,60 \\
\hline $\mathrm{R} \$ 253,97$ & 7 & 2 & 7 & 5 & $\mathrm{R} \$$ & $1.777,79$ & $\mathrm{R} \$$ & $1.269,85$ & $-R \$$ & 507,94 \\
\hline $\mathrm{R} \$ 170,16$ & 4 & 1 & 4 & 2 & $\mathrm{R} \$$ & 680,64 & $\mathrm{R} \$$ & 340,32 & $-R \$$ & 340,32 \\
\hline $\mathrm{R} \$ \quad 46,09$ & 2 & 1 & 2 & 2 & $\mathrm{R} \$$ & 92,18 & $\mathrm{R} \$$ & 92,18 & $\mathrm{R} \$$ & - \\
\hline $\mathrm{R} \$ 181,77$ & 0 & 0 & 0 & 1 & $\mathrm{R} \$$ & - & $\mathrm{R} \$$ & 181,77 & $\mathrm{R} \$$ & 181,77 \\
\hline $\mathrm{R} \$ \quad 47,07$ & 0 & 0 & 0 & 2 & $\mathrm{R} \$$ & - & $\mathrm{R} \$$ & 94,14 & $\mathrm{R} \$$ & 94,14 \\
\hline $\mathrm{R} \$ \quad 41,89$ & 0 & 0 & 0 & 1 & $\mathrm{R} \$$ & - & $\mathrm{R} \$$ & 41,89 & $\mathrm{R} \$$ & 41,89 \\
\hline $\mathrm{R} \$ 131,80$ & 0 & 0 & 0 & 1 & $\mathrm{R} \$$ & - & $\mathrm{R} \$$ & 131,80 & $\mathrm{R} \$$ & 131,80 \\
\hline $\mathrm{R} \$ 153,30$ & 7 & 4 & 7 & 6 & $\mathrm{R} \$$ & $1.073,10$ & $\mathrm{R} \$$ & 919,80 & $-R \$$ & 153,30 \\
\hline $\mathrm{R} \$ 155,79$ & 0 & 0 & 0 & 1 & $\mathrm{R} \$$ & - & $\mathrm{R} \$$ & 155,79 & $\mathrm{R} \$$ & 155,79 \\
\hline & & & & & RS & $6.709,94$ & RS & $10.552,25$ & RS & $3.842,31$ \\
\hline
\end{tabular}

Fonte: A autora (2020) 
Os cálculos foram feitos da seguinte maneira: Gasto para estoque correto é igual ao LEC multiplicado pelo Custo Médio Contábil, já o Valor Médio Investido em Estoque é igual ao Custo Médio Unitário vezes a 'Quantid'. Média Comprada desde a Abertura da Firma, e o Resultado é o Valor Médio Investido em Estoque menos o Gasto para estoque correto.

Ademais, como pode-se notar a empresa deveria ter investido $\mathrm{R} \$ 6.709,94$ na família de Bobinas de Ignição se os parâmetros de reposição de estoque tivessem sido feitos da maneira correta, usando o LEC, Entretanto, investiu-se R\$10.552,25, o que ocasionou um gasto desnecessário de $\mathrm{R} \$ 3.842,31$. Levando-se em conta as Bobinas de Ignição que nunca tiveram saída, portanto não deveriam ter sido compradas, a empresa gastou cerca de $\mathrm{R} \$ 2.161,84$, sendo que este valor poderia ter sido investido em produtos com maior demanda, aumentando possivelmente a receita para a empresa. Desta maneira, prova-se que não houve um planejamento nem um cálculo correto dos parâmetros de reposição de estoques por parte da gestão da empresa.

\section{CONCLUSÃO}

O objetivo principal da pesquisa foi apresentar novos parâmetros de reposição de estoques de produtos, neste caso especificamente a família de Bobinas de Ignição, escolhida através da aplicação do Teorema de Pareto, onde foi classificada como a família responsável por representar o maior faturamento médio, de 18,53\%, dentre as outras no mês de Agosto de 2020.

Após escolha, aplicou-se a filosofia do LEC (Lote Econômico de Compra) em que foi possível analisar que a empresa investiu, desde sua abertura, mais do que o necessário na compra de Bobinas de Ignição, onde foram investidos cerca de $\mathrm{R} \$ 10.552,25$. Se o parâmetro fosse seguido seria investido entorno de $\mathrm{R} \$ 6.709,94$, excedendo assim $\mathrm{R} \$ 3.842,31$ na compra de produtos indevidos.

De acordo com a pesquisa 15 diferentes tipos de Bobinas de Ignição, não deveriam ter sido compradas pois nunca tiveram saída e, a soma dessas compras é de cerca de R $\$ 2.161,84$. Concluindo-se que, realmente, não havia nenhum parâmetro a ser seguido para efetuar as compras dessas Bobinas, pois se seguida a filosofia abordada, o LEC, não haveria gastos desnecessários, compras indevidas e excesso de itens sem saída no estoque

Com o estudo foi possível apresentar um parâmetro ideal para que as tomadas de decisões a respeito das compras e reposições pudessem ser eficazes. Além disso recomenda-se um profissional capacitado para dar continuidade ao estudo para que ele possa fornecer informações, aplicando esses parâmetros para todos outros os itens que compõem o estoque, a 
fim de auxiliar a gestão no processo de tomada de decisão. Tendo, assim, potencial de diminuir o risco da empresa de adquirir produtos sem necessidades e/ou de não atender a demanda por falta dos mesmos. 


\section{APÊNDICE 01}

Faturamento por família de Produto Agosto 2020

\begin{tabular}{|c|c|c|c|}
\hline \multicolumn{4}{|c|}{ Faturamento por Família de Produto Agosto de 2020} \\
\hline Família de Produto & $\begin{array}{c}\text { Total de } \\
\text { Mercadoria }\end{array}$ & $\%$ & $\begin{array}{c}\% \\
\text { Acumulada }\end{array}$ \\
\hline BOBINAS DE IGNIÇÃO & RS $2.292,40$ & $18,5 \%$ & $18,5 \%$ \\
\hline CABOS DE IGNIÇÃO & RS $2.152,04$ & $17,4 \%$ & $35,9 \%$ \\
\hline LAMPADAS & RS $1.111,20$ & $9,0 \%$ & $44,9 \%$ \\
\hline VELAS DE IGNIÇÃO & RS 893,28 & $7,2 \%$ & $52,1 \%$ \\
\hline PISTÕES & RS 840,00 & $6,8 \%$ & $58,9 \%$ \\
\hline FILTROS DE AR & 760,20 & $6,1 \%$ & $65,1 \%$ \\
\hline AMORTECEDORES & RS 740,00 & $6,0 \%$ & $71,1 \%$ \\
\hline CORPOS DE BORBOLETA & RS 650,00 & $5,3 \%$ & $76,3 \%$ \\
\hline PALHETAS & 472,00 & $3,8 \%$ & $80,1 \%$ \\
\hline KITS EMBREAGEM & RS 361,18 & $2,9 \%$ & $83,0 \%$ \\
\hline COMANDOS DE VALVULA & RS $\quad 320,00$ & $2,6 \%$ & $85,6 \%$ \\
\hline SONDAS LAMBDA & 300,00 & $2,4 \%$ & $88,1 \%$ \\
\hline FUSIVEIS & 216,00 & $1,7 \%$ & $89,8 \%$ \\
\hline $\begin{array}{l}\text { REFIS BOMBA DE } \\
\text { COMBUSTIVEL }\end{array}$ & RS $\quad 179,50$ & $1,5 \%$ & $91,3 \%$ \\
\hline ЛUNTAS CABEÇOTE & RS $\quad 157,00$ & $1,3 \%$ & $92,5 \%$ \\
\hline PORTAS ESCOVA & RS $\quad 120,00$ & $1,0 \%$ & $93,5 \%$ \\
\hline BRONZINAS MANCAL & RS $\quad 100,00$ & $0,8 \%$ & $94,3 \%$ \\
\hline BOMBAS DE COMBUSTIVEL & 90,20 & $0,7 \%$ & $95,0 \%$ \\
\hline SENSORES DE NIVEL & 88,00 & $0,7 \%$ & $95,7 \%$ \\
\hline FARÓIS & 85,00 & $0,7 \%$ & $96,4 \%$ \\
\hline KIT DE AMORTECEDOR & 76,00 & $0,6 \%$ & $97,0 \%$ \\
\hline FILTROS DE COMBUSTIVEL & 60,00 & $0,5 \%$ & $97,5 \%$ \\
\hline PLATINADOS & 50,00 & $0,4 \%$ & $97,9 \%$ \\
\hline TAMPAS DE RESERVATÓRIO & RS $\quad 46,00$ & $0,4 \%$ & $98,3 \%$ \\
\hline BUCHA DA BANDEJA & 40,00 & $0,3 \%$ & $98,6 \%$ \\
\hline PIVOS DE SUSPENSÃO & 39,00 & $0,3 \%$ & $98,9 \%$ \\
\hline CABOS DE EMBREAGEM & 38,00 & $0,3 \%$ & $99,3 \%$ \\
\hline ROLAMENTOS DA RODA & 27,00 & $0,2 \%$ & $99,5 \%$ \\
\hline $\begin{array}{l}\text { ROLAMENTOS } \\
\text { ALTERNADOR }\end{array}$ & 26,00 & $0,2 \%$ & $99,7 \%$ \\
\hline RETENTORES & 23,43 & $0,2 \%$ & $99,9 \%$ \\
\hline FILTROS DE ÓLEO & 16,20 & $0,1 \%$ & $100,0 \%$ \\
\hline Total Geral & RS $12.369,63$ & $100,0 \%$ & $100,0 \%$ \\
\hline
\end{tabular}




\section{REFERÊNCIAS BIBLIOGRÁFICAS}

BALloU, RONALD H. Gerenciamento da Cadeia de Suprimentos: planejamento, organização e logística empresarial. 4. ed. Porto Alegre: Bookman, 2001.

BALLOU, R. H. Logística empresarial: transportes, administração de materiais e distribuição física. São Paulo: Atlas, 2007.

BITENCOURT, W. R. S.; CAVALCANTE, W. O. Determinação do Lote Econômico de Compras dos Produtos Classe A de uma Papelaria. 2013. Monografia (Bacharelado). Universidade da Amazônia. Belém. 2013.

BORGES C. T.; CAMPOS S. M.; BORGES C. E. Implantação de um sistema para o controle de estoques em uma gráfica/editora de uma universidade. Revista Eletrônica Produção \& Engenharia, v. 3, n. 1, p. 236 247, Jul./Dez.2010.

BOWERSOX, Donaldo J. Logística Empresarial: o processo de integração da Cadeia de Suprimento. São Paulo. Atlas, 2001.

CAMPOS, P. R. ; MARTINS, G. M. Administração de materiais e recursos patrimoniais. 1.ed. São Paulo: Saraiva, 2000. 335p

CHING, H. Y. Gestão de estoques na cadeia de logística integrada: supply chain. 4. ed. São Paulo: Atlas, 2010 .

CHOPRA, S. ; MEINDL, P. Gerenciamento da Cadeia de Suprimentos, Estratégia, Planejamento e Operação, São Paulo: Pearson, 2004.

DIAS, M. A. P. Administração de materiais: princípios, conceitos e gestão, 6 ed. São Paulo: Atlas, 2012.

MOREIRA, D. A. Administração da Produção e Operações. 1. ed. São Paulo: Pioneira, 1993.

SANTOS, A.M., RODRIGUES, I. A. Controle de Estoque de Materiais com Diferentes Padrões de

Demanda: Estudo de Caso em uma Indústria Química, Gestão \& Produção, vol. 13, n.2, pp.223-231, 2006.

SLACK, N.; CHAMBERS, S.; JOHNSTON, R. Administração da Produção. 3.ed. São Paulo: Atlas, 2009.

SOUZA, A. J. S.; BENTO, C. A.; MARTINS, R. Z.; LOPES, E. S.; GALVÃO, H. M. Análise da administração do capital de giro em uma empresa localizada na cidade de Guaratinguetá. Revista de Administração da Fatea.v.3, n.3, p.102-107, 2010.

TURBAN, E.; RAINER, R. K.; POTTER, R. R. Administração de Tecnologia da informação: teoria e prática. 2. ed. Rio de Janeiro: Elsevier, 2003.

WANKE, P. Gestão de Estoques na Cadeia de Suprimento: Decisões e Modelos Quantitativos. 1. ed. São Paulo: Atlas, 2003. 